\title{
Resolution of the Scanning Helium Microscope
}

\author{
S. Kalbitzer ${ }^{*}, 1$ and V. Zhukov ${ }^{2}$ \\ ${ }^{1}$ Ion Beam Technology, Heidelberg 69121, Germany \\ ${ }^{2}$ St. Petersburg Institute for Informatics and Automation of RAS, St. Petersburg 199178, Russia
}

\begin{abstract}
The resolution of helium ion scanning microscopes working in the secondary electron emission mode is theoretically estimated in the energy interval of $E=0.3-100 \mathrm{keV}$. The corresponding probe diameter improves with energy from 1.0 to $0.2 \mathrm{~nm}$. A theoretical probe diameter of $0.25 \mathrm{~nm}$ can be obtained by use of a standard three-electrode objective lens of electrostatic microscopes. The most important ion-optical element of this device is the supertip ion source. The existing devices, however, need calibration of spatial resolution. Three elementary types of test objects are suggested: multilayer nano-structures of metal on insulator, metal-phthalocyanine crystals, and "noise-like" objects such as metal nanoparticles on dielectric substrates. At low beam energies, a new type of contrast can be obtained in the mode of secondary electron registration with a resolution of about $1.1 \mathrm{~nm}$.
\end{abstract}

\section{INTRODUCTION}

During the last two decades a remarkable physical progress has taken place: The Müller field ion microscope, a device with atomic resolution [1], has been developed to an efficient ion source. To this end, ion emission from many atomic sites had to be confined to a single emitter point, as already proposed several decades ago [2]. Its experimental realisation, however, was not readily achieved [3]. The basic properties of a stable gas field ion source, GFIS, with a supertip, GFIS*, were first reported around 1990 [4].

The supertip is a nanocrystalline protrusion on a singlecrystal base tip. All rare gases and hydrogen can be used for generation of intense ion beams from a $\mathrm{W}$ emitter needle. Cryogenic temperatures, near the condensation point of the respective gas species, are recommended for maximum currents, of the order of $I^{+} \sim 1-10 \mathrm{nA}$ [4]. Currents of $I^{+}<1$ $\mathrm{nA}$, as obtained around the technologically more comfortable liquid nitrogen temperature, already suffice for many applications. Gas adsorption and desorption at the base tip, serving as an extended gas supply area, are the controlling mechanisms strongly competing with emitter tip temperature [5].

The excellent optical properties of a GFIS* source, with a typical half-angular spread $\alpha_{0} \sim 10 \mathrm{mrad}$ and virtual source size $d_{0} \sim 0.1 \mathrm{~nm}$, combine to an emittance figure $\varepsilon \sim 10^{-20} \mathrm{~cm}^{2} \mathrm{sr}$.

Thus, the corresponding brightness figures, $B=I / \varepsilon$, range up to $1 \mathrm{TA} / \mathrm{cm}^{2} \mathrm{sr}[6]$.

Consequently, by order of magnitude, nanometer image diameters can be predicted even for standard optical imaging

*Address correspondence to this author at the Ion Beam TechnologyConsulting, Bahofweg 2, 69121 Heidelberg, Germany;

E-mail: skalbitzer@aol.com systems, in absence of intra-beam and other broadening effects.

According to a frequently used approximation for chromatic limitations the spatial resolution reads $D_{c}=$ $2 C_{c}^{1 / 2} \eta^{1 / 2} \varepsilon^{1 / 4}$, where, in sequential order, we have the chromatic aberration coefficient, the relative energy spread, and the beam emittance [7]. By restricting ion beam emission angles, however, further improvements of $\varepsilon$ and hence of image diameters are feasible, at a concomitant expense of beam intensity, however.

As all focused ion beams remain practically nonbroadened close to the target surface, the emission of secondary electrons closely matches the beam image. It is estimated to be of the order of $D \sim 1 \mathrm{~nm}$, so that the ultimate resolution in surface analysis by secondary electron emission may also depend on this basic physical process and, therefore, should precisely be determined.

Recently, the engineering task of constructing a commercial helium ion microscope has successfully been carried out. Experimental resolutions of the order of $1 \mathrm{~nm}$ have been claimed by using focused $\mathrm{He}^{+}$beams for secondary electron imaging. Furthermore, the authors predict a theoretical probe diameter limit of $D \sim 0.25 \mathrm{~nm}$, or one atomic distance $[8,9]$. Thus, a broad field of application appears open for materials surface analysis far below the light-optical Abbé limit. Also, compared with conventional electron scanning technology, much better contrast is realised by helium beam scanning devices.

This device allows the use of two new contrast modes in the images of micro- and nano-objects $[10,11]$. These are Rutherford Backscattering (RBS) and Transmission Ion Microscopy (TIM). The first one allows the observation of so called Z-contrast in the images of target objects with different nuclear number [10].

However, as far as known to us, the publications of these authors lack pictures of any elementary etalon nano-objects 
of known sizes such as crystal lattice spacing etc. and also have no scale labels that correspond to sizes less than 200 nm.

Hence, the urgent question about both practical and theoretical resolution limits of a helium microscope remains to be answered. Thus, the purpose of this work is a theoretical estimation of the inherent potential ion-optical properties of such a device and the formulation of the requirements for determining the practical resolution and contrast of it.

\section{THEORETICAL ESTIMATION OF MINIMUM PROBE DIAMETER}

As well known, the square of the radius of a probe in scanning electron and ion microscopes is determined, basically, by aberrations of an objective lens and is given by the formula [12]:

$r_{P}^{2}=r_{G}^{2}+r_{C}^{2}+r_{D}^{2}+r_{S}^{2}$,

Here $r_{G}=r_{0} M$ is radius of the Gaussian image of the virtual ion source, $M$ is the linear magnification in the image of a source as determined by the Lagrange-Helmholtz theorem [12]:

$r_{0} \alpha_{0} \Delta E_{0}{ }^{1 / 2}=r_{G} \alpha_{p} E^{1 / 2}$,

where $\alpha_{0}$ is an aperture half angle of an ion beam in a source, $\Delta E_{0}$ is the $F W H M$ of the energy distribution in a source, $E=q U_{0}$ is the energy of ions incident onto the target, $q$ the ion charge, $U_{0}$ the accelerating potential, $\alpha_{p}$ the aperture half angle of the ion probe on a target.

So, from Eq. (2) we have $M=r_{G} / r_{0}=\eta^{1 / 2} \alpha_{0} / \alpha_{p}$, where $\eta=\Delta E_{0} / E$ is the relative energy spread. According to [10], at $E=30 \mathrm{keV}$ the relation holds: $\eta=2 \cdot 10^{-5}$, so $\Delta E_{0}=0.6 \mathrm{eV}$. In Eq. (1) $r_{C}=C_{C} \eta \alpha_{p} / 2$ is the radius of the chromatic aberration circle, $C_{C}$ the coefficient of the chromatic aberration, $r_{p}=0.61 \lambda / \alpha_{p}$ the radius of the diffraction error circle in the Rayleigh approximation. $\lambda=h(2 m E)^{-1 / 2}$ is the de Broglie wavelength of the ion at target position, $h$ is Planck's constant, $m$ the ion mass. $r_{S}$, the radius of the spherical aberration disk, is negligibly small in comparison with other contributions in Eq. (1) [12].

Substituting in Eq. (1) these definitions, neglecting $r_{S}$, the probe radius as a function of the aperture half angle $\alpha_{p}$ reads:

$r\left(\alpha_{p}\right)=\left[r_{0}^{2}\left(\alpha_{0} / \alpha_{p}\right)^{2} \eta+C_{C}^{2}(\eta / 2)^{2} \alpha_{p}^{2}+\left(0.61 \lambda / \alpha_{p}\right)^{2}\right]^{1 / 2}$,

The minimum of this function is reached at

$\alpha_{p}=1.105\left(C_{c} \eta\right)^{-1 / 2}\left(\lambda^{2}+2.687 r_{0}^{2} \alpha_{0}^{2} \eta\right)^{1 / 4}$,

and equals

$r_{\min }=0.773\left(C_{c} \eta\right)^{1 / 2}\left(\lambda^{2}+2.687 r_{0}^{2} \alpha_{0}^{2} \eta\right)^{1 / 4}$.

Traditionally, in electrostatic microscopes the threeelectrode one-potential lens, "Einzel-lens", with a thick cen- tral electrode $[12,13]$ is used as an objective lens. This lens is shown in Fig. (1).

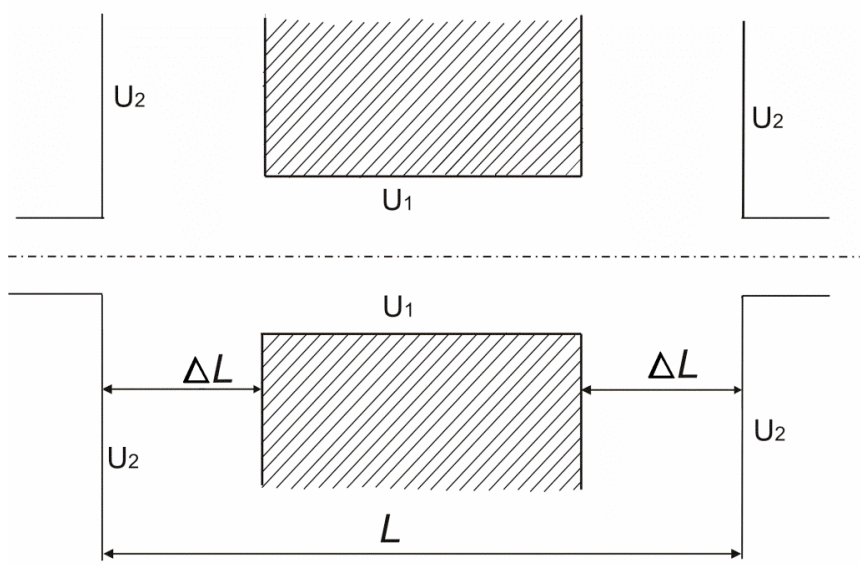

Fig. (1). Potential scheme of an Einzel-lens with a thick central electrode. Here $L$ is the distance between the outer electrodes, $\Delta L$ the distance between the central and the outer electrodes, $U_{I}$ the potential of the central electrode, $U_{2}$ the potential of the outer electrodes.

As $C_{C} \propto L$, where $L$ is the length of a lens [12], in practice a tendency exists to reduce the size of $L$.

At the given differences of potentials $\Delta U=U_{1}-U_{2}$ between the central electrode and the outer electrodes of a lens the size $\Delta L$ of intervals between electrodes is limited from below so that the value $\Delta U / \Delta L$ is less than the value of the maximal strength of an electric field corresponding to socalled electrical breakdown in vacuum: maximum of $\Delta U / \Delta L$ is less than $15 \mathrm{kV} / \mathrm{mm}[12,13]$. Hence, size $L$ is limited from below too. It is necessary to note that at given $L$ the relation holds: $C_{C} \propto f$ [12], where $f$ is the focal length of a lens. The focal length in turn is determined by the working distance $(W D)$ of the objective lens usually defined as the distance between the last electrode and the target. WD depends mainly on the thickness of the secondary particle detector and on both size and tilt of the specimen. The relation $f \geq W D$ must be fulfilled. Let's find out, how the size of a probe diameter $d_{p}=2 r_{\min }$ will change depending on the ion energy on a target $E$ under the condition $\max (\Delta U / \Delta L) \leq 15 \mathrm{kV} / \mathrm{mm}$. As a starting point of this research we will use the following data:

a) In [11] the 'Essential Specification' data of helium microscope is given as $W D=4 \mathrm{~mm}$.

b) In [8-11] energy of ions $E$ is changing in the interval $10-45 \mathrm{keV}$.

We shall take the size $W D=4 \mathrm{~mm}$ and $E$ at an intermediate point of the above mentioned interval. For example, let $E$ be $25 \mathrm{keV}$. We choose the distance between the central electrode and the outer electrodes, and also differences of potentials between them so that the considered three-electrode lens allows the minimal value of coefficient $C_{C}$ at energy $E=25 \mathrm{keV}$ and would have the size $W D=4 \mathrm{~mm}$ [11]. Our 
calculation gives the values: $C_{C}=17 \mathrm{~mm}$ and $d_{p}=0.32 \mathrm{~nm}$ at $\Delta E_{0}=0.6 \mathrm{eV}$.

Let's change now energy of ions $E$ in an interval 5-100 $\mathrm{kV}$ and also proportional to all geometrical sizes of a lens so that the condition is fulfilled: $\max (\Delta U / \Delta L) \leq 15 \mathrm{kV} / \mathrm{mm}$.

In Fig. (2) the diagrams of dependence of sizes $\eta / 2, W D$, $C_{C}$ and $d_{p}$ from change of energy of ions $E$ in an interval 5$100 \mathrm{kV}$ and at $\Delta E_{0}=0.6 \mathrm{eV}$ are shown.

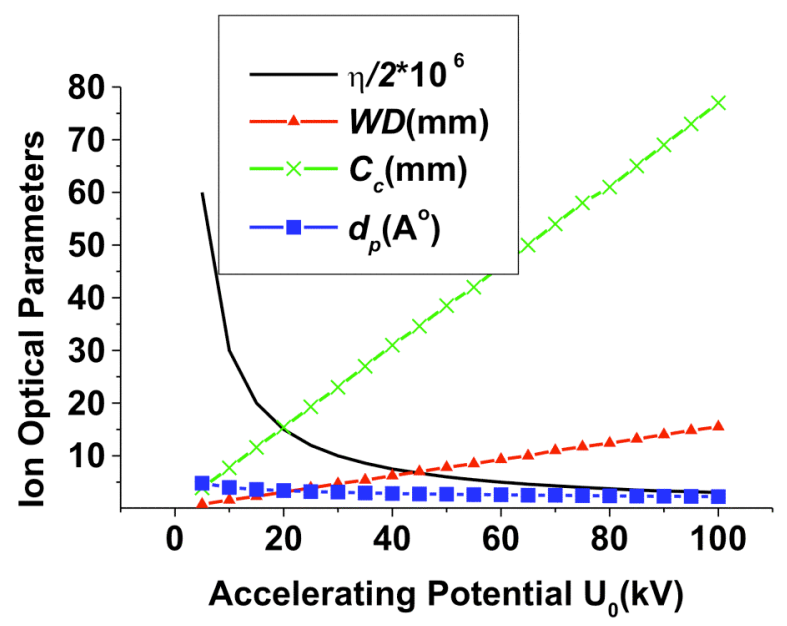

Fig. (2). The dependence of ion optical parameters on the acceleration potential $U_{0}$ in high energy interval. Here $\eta=\Delta E_{0} / E$ is the relative energy dispersion of the ion beam, $W D$ the working distance of the objective lens, $C_{C}$ the chromatic aberration coefficient, $d_{p}$ the probe diameter on the target.

From this diagram it appears, that $C_{c}$ and $W D \propto E$. Taking into account the dependence on energy of values $\lambda \propto E^{-1 / 2}$ and $\eta \propto E^{-1}$, we shall obtain: $d_{p} \propto E^{-1 / 4}$. This very weak dependence of the image spot size on the beam energy is quite remarkable and will be illustrated for a few chosen cases.

At $E=70 \mathrm{keV}$, we find $d_{p}=0.25 \mathrm{~nm}$ and $W D \sim 13 \mathrm{~mm}$. Taking a variant of the lens geometry corresponding to $E=30 \mathrm{keV}$, and slightly increasing the potential on the central electrode (under condition $\max (\Delta U / \Delta L) \leq 15 \mathrm{kV} / \mathrm{mm}$ ), resulting in $W D=4 \mathrm{~mm}$ (instead of $W D \sim 5 \mathrm{~mm}$ according to Fig. (2)), we obtain for the objective lens $C_{C}=15 \mathrm{~mm}$ and $d_{p}=0.25 \mathrm{~nm}$.

Taking another variant of the lens geometry corresponding to $E=45 \mathrm{keV}$ and also slightly increasing the potential on the central electrode, resulting in $W D=4 \mathrm{~mm}$ instead of $W D=10 \mathrm{~mm}$, we obtain for the objective lens $C_{C}=27 \mathrm{~mm}$ and $d_{p}=0.24 \mathrm{~nm}$.

At the extreme energy of $E=100 \mathrm{keV}$, by slightly increasing the potential on the central electrode, with the result of $W D=4 \mathrm{~mm}$ instead of $W D=15 \mathrm{~mm}$, we obtain for the objective lens $C_{C}=50 \mathrm{~mm}$ and $d_{p}=0.18 \mathrm{~nm}$.

Thus, the theoretical value of the minimal probe diameter, equal to $2.5 \AA$ as declared in work [9], is not extreme and can be realized with a standard three-electrode electrostatic objective lens.

The most extreme ion-optical element of this microscope is its 'supertip' ion source that was suggested and developed in $[4,5,6]$. This source possesses unique small size and allows to use a telescopic two-lens ion optical system without strong demagnification of a source. Magnification changes between $M=0.33$ and $M=0.07$ with the ion energy in the interval $E=5-100 \mathrm{keV}$. A telescopic two-lens ion optical system in turn provides the possibility of using an objective lens in the mode of so called infinitely small magnification where the chromatic aberration coefficient is extremely small [12]. An ion probe diameter of $0.25 \mathrm{~nm}$, however, is an "unobservable" physical parameter until a method for measuring such small probes will have been developed.

Note that the resolution $D$ in a helium microscope is determined not only by the probe diameter, but also by the iontarget interaction, so the formula applies:

$D=\sqrt{d_{p}^{2}+d_{t a r}^{2}}$,

where $d_{t a r}$ is the diameter of the secondary emission area by irradiation of a target with an infinitely thin ion beam.

Unfortunately, the practical resolution in a helium microscope is remaining unknown so far. The images of the objects considered in [9-11], lack scale labels of spacing less than $200 \mathrm{~nm}$ as well as images of any elementary test-object of precisely known atomic size.

\section{PROPOSED CALIBRATION OF PRACTICAL RESO- LUTION}

Already for more than 30 years, elementary test-objects have been used for calibration of high resolution transmission electron microscopes (TEM). This test-objects exhibit either precisely known sizes (crystals) or a constant spectral density in the area of relevant spatial frequencies ("noise like" objects such as a thin amorphous carbon films) [14, 15].

Thus, for the calibration of a helium microscope by analogy to electron microscopy, we suggest the use of testobjects containing the necessary spatial periods (frequencies). In Fig. (3), a composite structure with alternating layers of metal and dielectric at periods of $2 \mathrm{~nm}$, obtained by the method of molecular (or atomic) beam epitaxy (MBE), is schematically represented. In this structure $2 \mathrm{~nm}$-layers of tungsten alternate with those of silicon dioxide [16, 17]. (a)

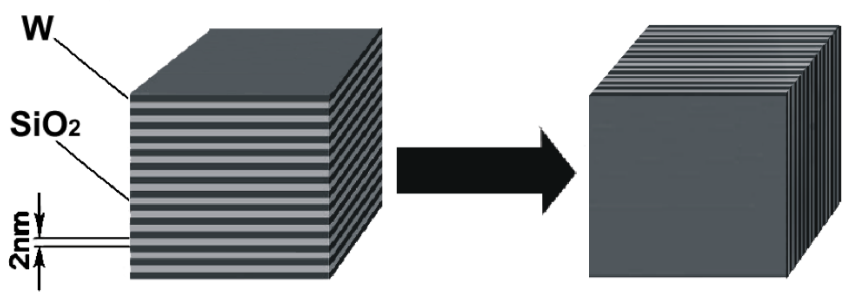

Fig. (3). Illustration of composition (a) and application (b) of a multilayer target for measurement of spatial resolution in scanning ion microscopy. 
In another approach, $\delta$-layers of $\mathrm{Sb}$ in a $\mathrm{Si}$ matrix were used to define the precise target geometry for ion beam stopping experiments [18].

Obtained with an ion microscope with a Liquid Metal Ion Source (LMIS) the cross-section of such structures $[16,17]$ could be used for an initial calibration of helium microscopes. The technology of obtaining such layers is mastered in [16-18]. According to [10], metals and isolators give essentially different contrast in helium microscope in the secondary electron emission mode. Such structures will well be visible at a resolution of $5 \AA$.

As a second test object a molecular metal-organic crystal $[19,20]$ as presented schematically by Figs. (4-6). Fig. (4) presents the elementary cell of this crystal, one molecule of Fe-phthalocyanine. In Fig. (5) a small fragment of this molecular crystal is shown with a scale label. From this picture it can be seen that in a crystal of Fe-phthalocyanine the distance between atoms of metal (in this case iron) is equal to $16 \AA$. Fig. (6) presents a larger fragment of it.

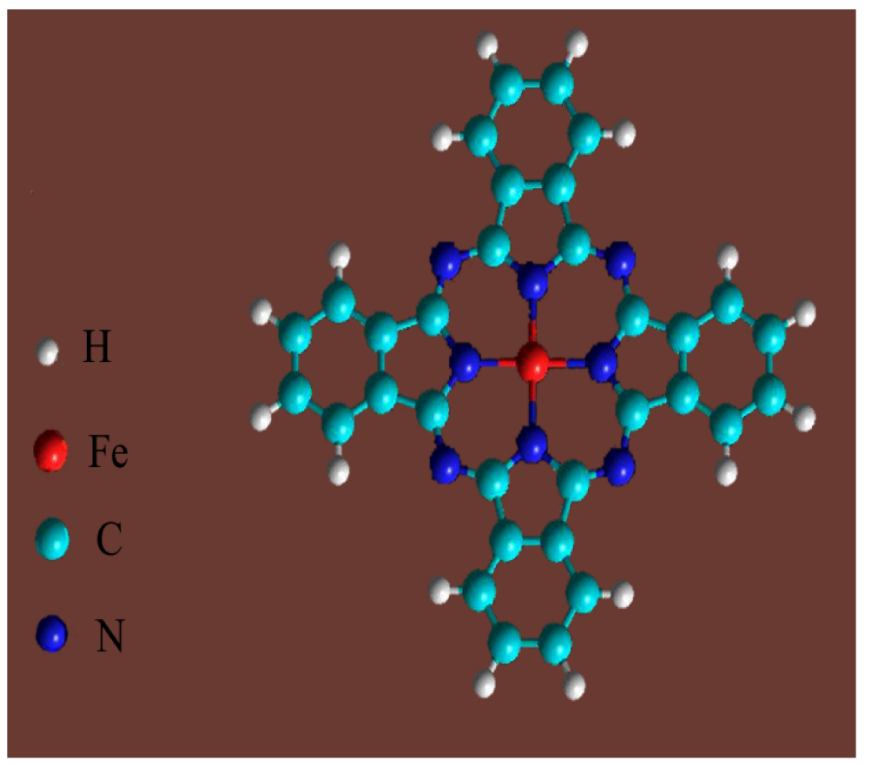

Fig. (4). The schematic structure of a Fe-Phthalocyanine molecule, the elementary cell of this metal-organic crystal.

It is expected that the emission of secondary electrons from the lines of metal atoms will be much higher than from surrounding organic molecules.

For the third type of tests-objects for helium microscopes as suggested in [21], "noise-like" structures consisting of various sizes sprayed on a dielectric could serve.

\section{SPECTRAL ANALYSIS OF SPATIAL FREQUENCIES IN IMAGES}

Because of the absence of images of elementary testobjects in Refs. [8-11], we have undertaken an attempt to define the actual resolution with use of a spectrum analysis method for the spatial frequencies presented [21]. According to [21], only images with the known field of view (FOV) or with scale labels and with known number of pixels are

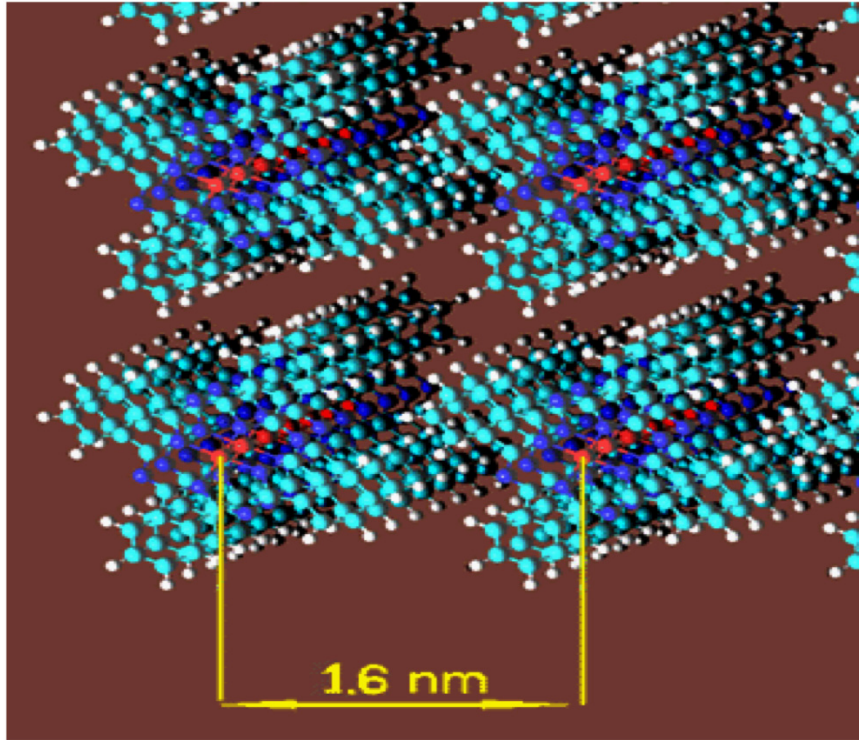

Fig. (5). A small fragment of molecular crystal with scale label.

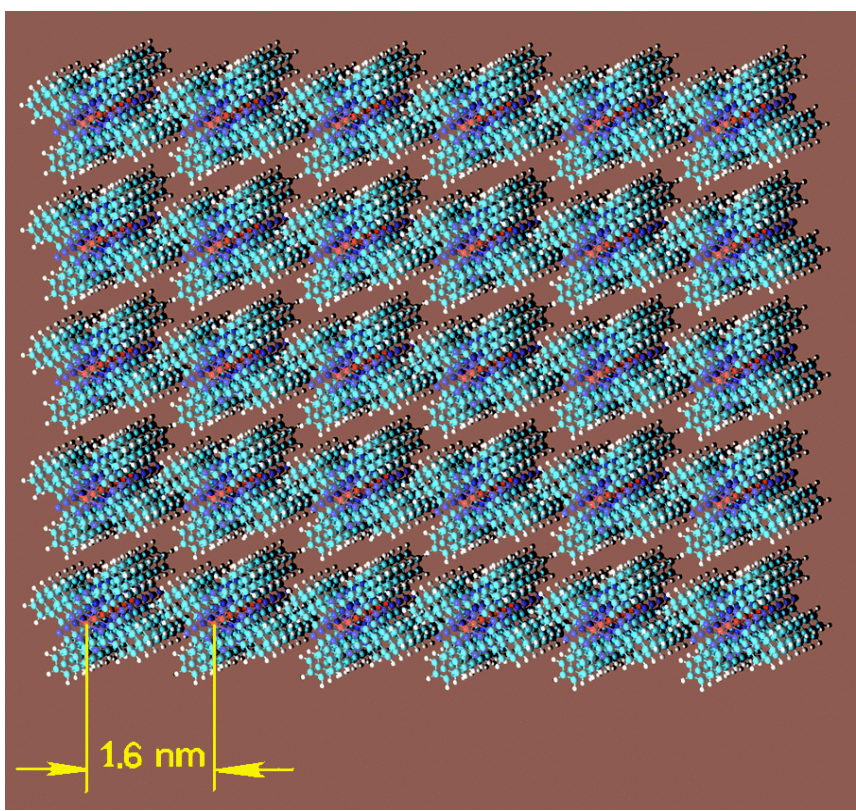

Fig. (6). A large fragment of molecular metal-organic crystal.

suitable for this aim. In Refs. [8-11] we could find only one such image. It is the rough image of an amorphous object from Ref. [10], Fig. (15), with the FOV equal to $50 \mu \mathrm{m}$ containing $1400 \times 1400$ pixels. So the spatial periods that are less than $50 / 1400 \mu \mathrm{m}(\sim 36 \mathrm{~nm})$ must be absent in this image. To prove the usability of above mentioned method this image was converted to a numerical file, and after that the 1D Fourier-transformations of the obtained 2D matrix was made line-by-line.

Fig. (7) presents the power spectrum averaged over all lines of a matrix and normalized to the amplitude of spatial frequency of constant background. As visible from this diagram the spectral density of spatial periods, smaller than 150 nanometers, contributes less than $1 \%$ referred to a constant background level. 


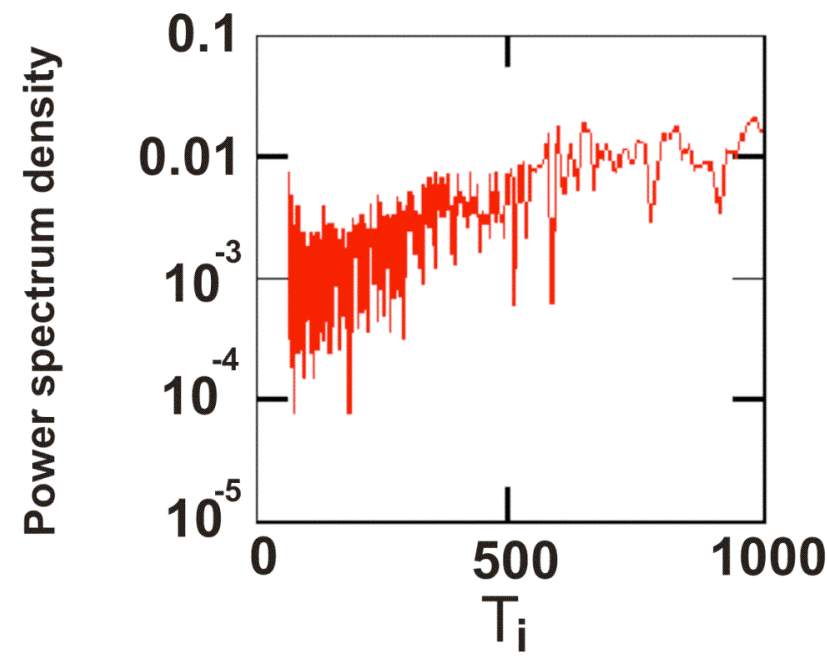

Spatial period in $\mathrm{nm}$

Fig. (7). 1D power spectrum of image from [10] averaged over all lines of a 2D matrix of 1400x1400 pixels.

Thus, the displayed object does not present any fine details less than $50 / 1400 \mu \mathrm{m}$ and no small false spatial period with statistical significant weight did appear as a result of spectrum analysis. A two-dimensional power spectrum of spatial frequencies for the same image [10] is presented in Fig. (8). From this picture it is visible that $2 \mathrm{D}$-spectrum is isotropic as it must be for amorphous objects and the FWHM of the spatial spectrum is equal approximately to $0.01 \mathrm{~nm}^{-1}$ that corresponds to spatial period of $100 \mathrm{~nm}$. Only an insignificant amount of single points that correspond to the above mentioned theoretical limit spatial period of $\sim 36 \mathrm{~nm}$ are present also.

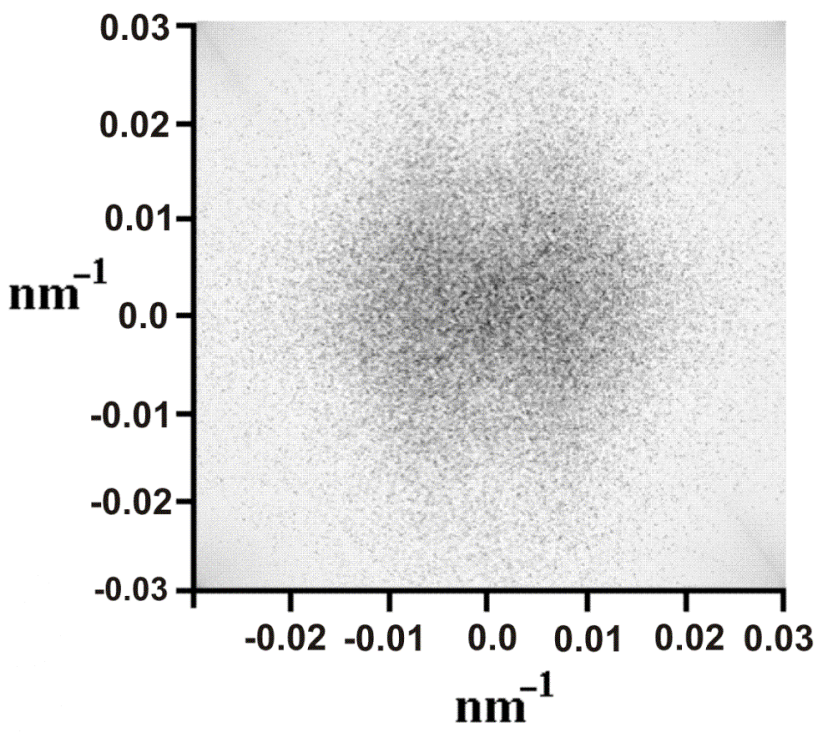

Fig. (8). The 2D power spectrum of the image from work [10].

\section{AN ADDITIONAL CONTRAST MODE}

As mentioned above, in a helium microscope two new kinds of contrast, RBS and TIM mode, are realized. According to $[22,23]$, another kind of contrast can be utilized as well. It is connected to the potential secondary emission of electrons from clean metal surfaces occurring at low beam energies of $E \sim 0.3 \mathrm{keV}$.

In Fig. (9) a continuation of Fig. (2) in the regime of low beam energies is presented. Apparently, at an energy of $\sim 0.3$ $\mathrm{keV}$ it is possible to obtain a probe diameter of $d_{p} \sim 1 \mathrm{~nm}$ simply by use of a standard three-electrode lens.

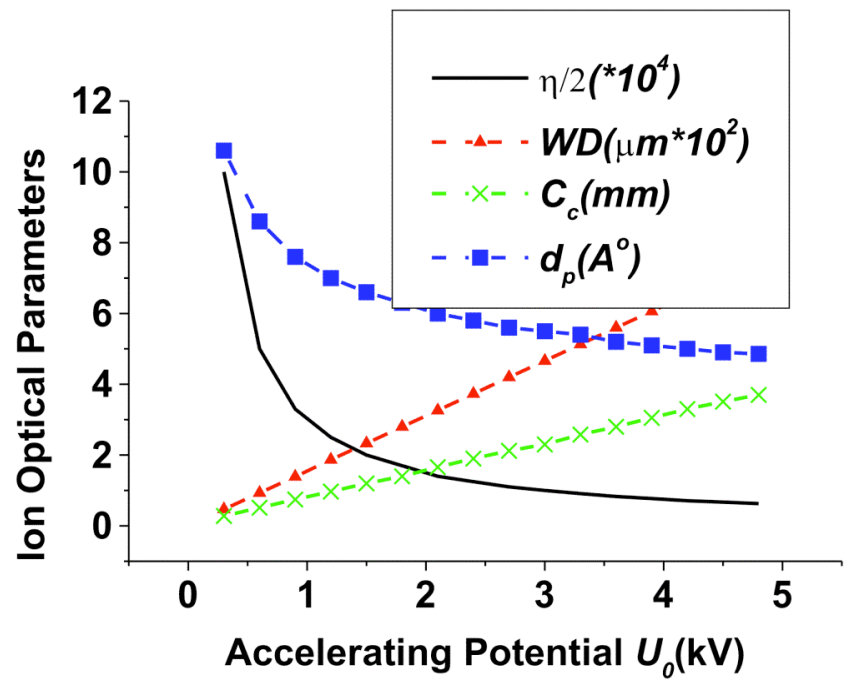

Fig. (9). Dependence of ion optical parameters on the acceleration potential $U_{0}$ in the low energy regime. Here $\eta=\Delta E_{0} / E$ is the relative energy dispersion of the ion beam, $W D$ the working distance of objective lens, $C_{c}$ the coefficient of chromatic aberration, $d_{p}$ the probe diameter on the target.

Consider the case of an analytical microscope using lowenergy $\mathrm{He}^{+}$ions and the existing conditions for its implementation. As is well known [24, 25], helium has the greatest first ionization potential of all chemical elements, equal to $24.5 \mathrm{eV}$. Because of this a $\mathrm{He}^{+}$ion with an arbitrarily small kinetic energy is capable of exciting potential secondary electron emission with a yield factor of about $30 \%$ when striking a metal surface. For most pure metals, the excitation threshold of kinetic secondary electron emission is higher than $400 \mathrm{eV}$ [24]. To deal with pure potential emission, we assume that the energy $E$ of helium ions at the target is $300 \mathrm{eV}$. Today, the phenomenon of potential secondary electron emission from a metal surface exposed to lowenergy $\mathrm{He}^{+}$ions is being used to obtain more information on the arrangement of surface energy levels [25]. It is therefore natural to try to use this effect also for studying the spatial structure of nanoscale metal objects.

It is now held that the excitation of secondary potential emission of electrons by a $\mathrm{He}^{+}$ion involves the three-step process represented schematically in Fig. (10) [22, 25]:

the transformation of the potential threshold at the metal surface into a potential barrier under the action of an incident $\mathrm{He}^{+}$ion;

(ii) the tunnel transition of an electron (1) from the conduction band of the metal to the incident $\mathrm{He}^{+}$ion through the vacuum potential barrier with the formation of an excited neutral He atom; 
(iii) the transfer of this excitation to an electron (2) in the conduction band and the Auger transition of this electron (2) into the vacuum through a residual potential barrier.

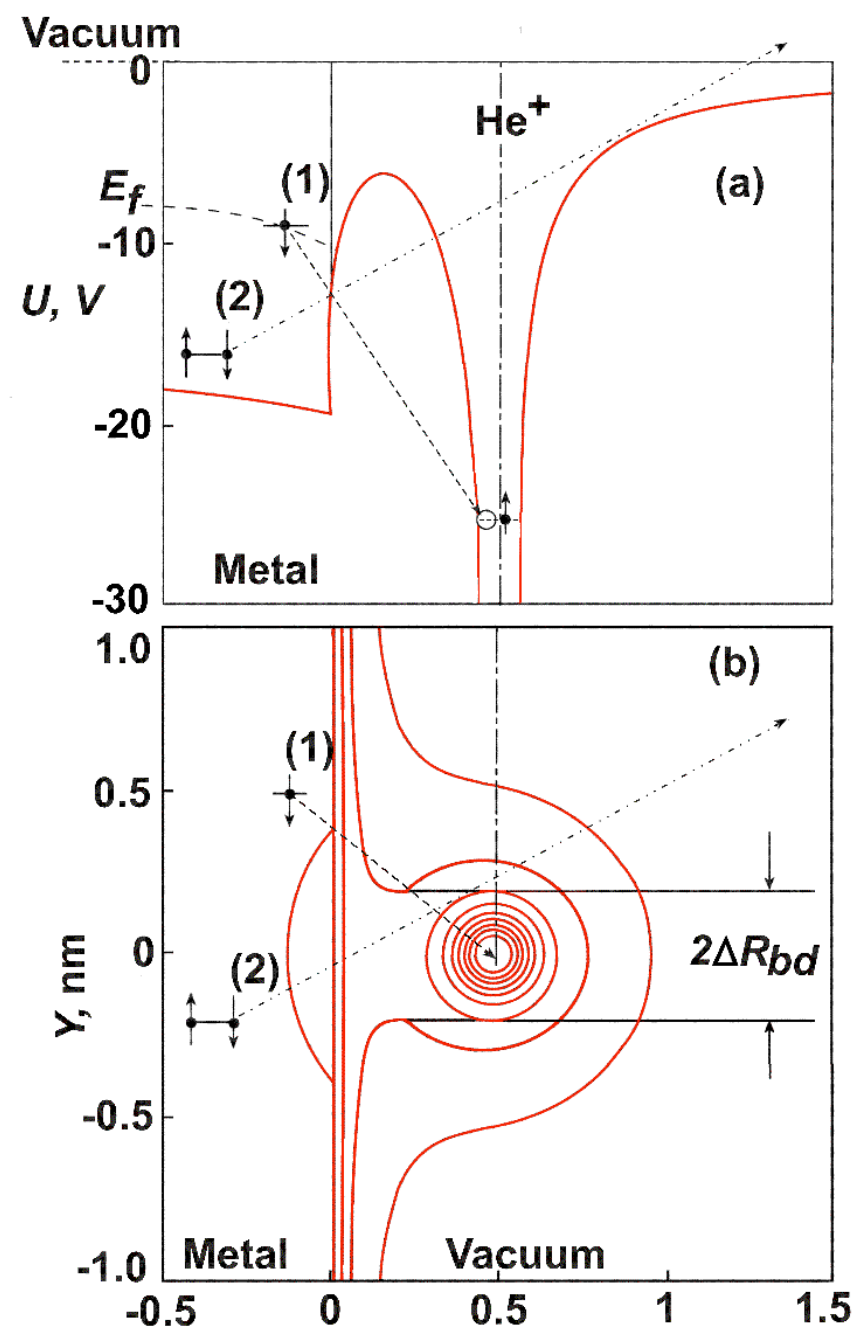

Fig. (10). (a) Diagram with coordinates $\{X, U\}$, where $x$ is the distance along the $X$ axis perpendicular to the crystal surface and passing through the center of a $\mathrm{He}+$ ion, $U$ the potential in volts relative to the vacuum level. (b) Equipotential pattern on the plane $\{X, Y\}$, where the $X$ axis is as in panel (a) and the $Y$ axis passes along the metal surface; the distance in nanometers is plotted along the axes $\{X, Y\}, \Delta R_{\mathrm{bd}}$ is the radius of the deformation region of the potential barrier at the metal-vacuum interface.

The entire process of Auger-electron emission ends before the helium projectile comes to rest beneath the solid surface. Thus, $d_{t a r}$ in Eq. (6) represents the diameter of the area of potential secondary electron emission for an infinitely thin ion beam incident on a target. This size is equal to the diameter $2 \Delta R_{b d}$ of the surface area where the abovementioned transformation of the potential threshold occurs (Fig. 10b). According to [25], $d_{t a r}=2 \Delta R_{b d} \approx 0.5 \mathrm{~nm}$. Substituting in Eq. (6) $d_{t a r}=0.5 \mathrm{~nm}$ and $d_{p}=1 \mathrm{~nm}$ it appears theoretically possible to achieve a resolution of $1.1 \mathrm{~nm}$ in this mode. However, the working distance of a lens at energy $E=0.3 \mathrm{kV}$ is only $50 \mu \mathrm{m}$. Therefore, there is a problem of development of a secondary electron detector with the thick- ness of approximately $25 \mu \mathrm{m}$. However, according to [26], this problem can be solved.

On the other hand, if an ion-optical resolution limit of $d_{p}$ $=0.25 \mathrm{~nm}$ is realized, the target effect of $d_{\text {tar }}=0.5 \mathrm{~nm}$ would limit the actual resolution roughly to $D \sim 0.6 \mathrm{~nm}$.

\section{CONCLUSIONS}

Apparently, a simple two-lens ion optical system for a helium ion microscope is feasible. A theoretical probe diameter of $0.25 \mathrm{~nm}$ can be obtained by means of standard three-electrode objective lenses as in use with electrostatic microscopes.

An ion probe diameter of $0.25 \mathrm{~nm}$, however, is an "unobservable" physical parameter until a method for measuring such small probes will have been developed.

The most important ion-optical element of a helium microscope is the supertip ion source. It allows the use of a telescopic two-lens ion optical system with moderate demagnification of the source geometry and small coefficients of chromatic aberration.

However, in view of the high discrepancy between a theoretical resolution of $D<1 \mathrm{~nm}$ and the actual experimental observations of $D>36 \mathrm{~nm}$ it must be concluded that strong additional noise contributions exist, be it by vibration and/or misalignment of the emitting supertip. (See paragraph 4 of present paper).

Therefore the presently existing helium microscope urgently needs calibration of the achieved resolution by suitable elementary etalon test-objects, for example, by testobjects suggested in the present paper, paragraph 3 .

After that, the helium ion microscopy may serve as a defined tool for an unprecedented precise analysis of nanoobjects.

\section{REFERENCES}

[1] Müller EW. Das Feldionenmikroskop. Z Physik 1951; 131: 136-42.

[2] Gomer R. Field emission and field ionisation. Harvard University Press: Cambridge; 1961.

[3] Schwoebel PR, Hanson GR. Beam current stability from localized emission sites in a field ion source. J Vac Sci Technol B 1985; 3 : 214-19.

[4] Böhringer K, Jousten K, Kalbitzer S. Development of a highbrightness gas field-ionization source. Nucl Instr Meth Phys Res B 1988; 30: 289-92.

[5] Kalbitzer S. Generation of optical contrast in insulating materials by irradiation with focused ion beams. Appl Phys A 2000; 71: 5659.

[6] Kalbitzer S, Wilbertz CH, Miller TH, Knoblauch A. Ion beam modification for submicron technology. Nucl Instr Meth Phys Res B 1996; 113: 154-60.

[7] Legge GJF. The limits of spatial resolution in pixe. Nucl Instr Meth Phys Res B 1987; 22: 115-20.

[8] Notte J, Hill R, McVey S, Farkas L, Percival R, Ward B. An Introduction to Helium ion microscopy. Microsc Microanal 2006; 12(Suppl S02): 126.

[9] Ward BW, Notte JA, Economou NP. Helium ion microscope: A new tool for nanoscale microscopy and metrology. J Vac Sci Technol B 2006; 24(6): 2871-74.

[10] Morgan J, Notte J, Hill R, Ward B. An Introduction to the helium ion microscope. Microse Today 2006; 14(4): 24-31.

[11] Essential Specification data of "ORION" helium ion microscope Available from: http://www.smt.zeiss.com/nts, July 27, 2008. 
[12] Szilagyi M. Electron and ion optics. Plenum Press: New York; 1988.

[13] Grivet P. Electron optics. Pergamon Press: New York; 1965.

[14] Heidenreich R. Fundamentals of Transmission Electron Microscopy. Interscience Publisher: New York, London, Sydney; 1964.

[15] Spense J. Experimental high resolution electron microscopy. Clarendon Press: Oxford; 1981

[16] Driskill-Smith AAG, Hasko DG, Ahmed H. The "nanotriode:" A nanoscale field-emission tube. Appl Phys Let 1999; 75(18): 284547.

[17] Driskill-Smith AAG, Hasko DG, Ahmed H. Quantum interference in vacuum nanotriode. J Vac Sci Technol B 2000; 18(6): 3481-87.

[18] Niemann D, Oberschachtsiek P, Kalbitzer S. Energy loss and strggling of $\mathrm{MeV}^{4} \mathrm{He}$ ions in a $\mathrm{Si} / \mathrm{Sb}$ multilayer target. Nucl Instr Meth Phys Res B 1993; 80-81: 37-40.

[19] Engel MK, Kawamura RKH. Single-Crystal and Solid-State Molecular Structures of Phthalocyanine Complexes. 1996. Available from: http://phthalo.mkengel.de/KICReview.pdf, July 27, 2008.

[20] Li-Ying C, Yang J, Fu Q, Bao-Zhong Z, Tian L, Hai-Ling Y. Synthesis, crystal structure and characterization of a new zinc phthalocyanine complex. J Mol Struct 2007; 827(1): 149-54.
[21] Orloff J, Swanson LW, Utlaut M. Fundamental limits to imaging resolution for focused ion beams. J Vac Sci Technol B 1996; 14(6): 3759-63.

[22] Zhukov VA, Titov AI, Zavyalova AV. Using a chromaticaberration correction system to achieve sub-1.6-nm resolutions of a focused-ion-beam microscope designed for characterization and processing. Russian Microelectronics 2007; 36 (6): 279-87.

[23] Zhukov VA. Achieving sub-1.6-nm resolutions of a low-voltage microscopic focused-ion-beam system not involving aberration correction. Russ Microelec 2008; 37(2): 98-106.

[24] Petrov NN, Abroyan I. Diagnostika poverkhnosti s pomoshchyu ionnykh puchkov (ion-beam characterization of surfaces). Nauka: Leningrad; 1977.

[25] Cazalilla MA, Lorente N, Diez MR. Theory of auger neutralization and deexcitation of slow ions at metal surfaces. Phys Rev B 1998; 58(20): 13991-4006.

[26] Schenkel T, Persaud A, Park SJ, et al. Single ion implantation for solid state quantum computer development. J Vac Sci Technol B 2002; 20(6): 2819-23.

Received: May 16, 2008

Revised: July 10, 2008

Accepted: July 14, 2008

(c) Kalbitzer and Zhukov; Licensee Bentham Open.

This is an open access article licensed under the terms of the Creative Commons Attribution Non-Commercial License (http://creativecommons.org/licenses/by$\mathrm{nc} / 3.0 /$ ) which permits unrestricted, non-commercial use, distribution and reproduction in any medium, provided the work is properly cited. 